\title{
L'évaluation en classe de FLE : les représentations sociales qu'en ont certains professeurs au Mexique
}

\author{
Noëlle Groult \\ Centre d'Enseignement de Langues Etrangères, Université National Autonome du Mexique \\ groult@servidor.unam.mx
}

\section{Introduction}

Que nous le voulions ou pas, nous passons souvent le filtre d'une évaluation, de ce que nous faisons, de ce que nous pensons ou disons, au travail ou dans le contexte de notre vie au quotidien. Evaluer implique une tâche complexe, par les éléments cognitifs, sociaux, psychologiques et culturels que cela met en cause, et ce n'est pas toujours perçu favorablement, ni par les évaluateurs ni par ceux qui sont évalués; la salle de classe en est un bon exemple car souvent la mise en place des modalités d'évaluation ne sont pas claires, posent problème et les résultats n'en sont pas toujours acceptés de bon gré.

Par ailleurs, suivant la théorie développée par Moscovici (1961), il est accepté que nous avons tous des représentations sociales sur les sujets les plus divers et que, d'une part, celles-ci nous aident à comprendre, à structurer, à apprivoiser la réalité qui nous entoure et que, d'autre part, elles peuvent expliquer notre manière d'agir et de réagir face aux activités quotidiennes, aux événements inattendus, aux problèmes qui se présentent à nous. Elles servent aussi à la construction de l'identité des individus d'un groupe face à d'autres. Elles se construisent à partir de différents mécanismes mais en premier lieu grâce à la communication en société, face à des phénomènes qui provoquent des tensions ou viennent changer le contexte auquel nous sommes habitués.

De par l'importance de l'évaluation dans l'enseignement ainsi que des représentations sociales en général, nous avons entrepris une recherche au Département de Français du Centre d'Enseignement de Langues Etrangères (CELE) de l'Université Nationale Autonome du Mexique (UNAM) dans le cadre d'un Doctorat en Pédagogie de la Faculté de Philosophie et Lettres de la même Université. Notre objectif était de détecter les représentations sociales des professeurs de FLE sur l'évaluation en salle de classe, c'est à dire, comment ils évaluent les compétences en FLE de leurs élèves: au niveau de la compréhension et production, la grammaire, le lexique, l'efficacité communicative; nous voulions aussi découvrir leur opinion sur les modalités d'évaluation proposées (examens partiels et de fin de semestre) par leur Département; de même nous voulions vérifier s'il existait ou non des coïncidences entre les représentations des professeurs et ces modalités d'évaluation et si les unes et les autres avaient une influence sur leurs pratiques en classe.

\section{La théorie des représentations sociales (TRS)}

La théorie des représentations sociales (TRS) peut être très utile quand on essaie de décrire et de comprendre les conduites dans différents contextes de l'activité humaine. Depuis 1961, date à laquelle Moscovici l'a formulée dans son livre "La Psychanalyse, son image et son public», elle a été prise comme cadre de référence dans bien des recherches en sciences humaines, sociologie, anthropologie, psychologie sociale, etc. Pour Moscovici (1984), les représentations sociales sont des processus mentaux qui aident à organiser la réalité physique et imaginaire qui conforme la réalité sociale. Sous-jacente à tout le concept, on trouve la notion de sens commun qui est une manifestation de connaissance spécifique, qui reflète la mise en marche de 
processus socialement fonctionnels. Les représentations sociales établissent une relation entre quelqu'un, ou un groupe, et quelque chose. De là, que l'interaction et la communication sociale soient des éléments primordiaux dans cette construction de l'autre et qu'il y intervienne aussi des éléments symboliques, affectifs et cognitifs.

Les représentations sont sociales car elles sont produites par une collectivité, sans que l'on puisse ignorer le fait que chaque individu, ses relations interindividuelles et réactions face aux impositions sociales ont un certain potentiel de création. Les représentations se localisent dans l'imaginaire collectif et trouvent leur sens et cohérence à partir des consensus, valeurs et normes que la culture impose. Elles contribuent à la formation, orientation et expression des conduites et communications sociales. Elles se construisent et reconstruisent, selon des processus de signification et re-signification par le truchement de l'expérience.

Les représentations sociales rendent familier et acceptable ce qui nous est inconnu, par l'intermédiaire de la mémoire, le passé, l'image. Selon Abric (1994), elles ont une fonction de savoir car elles aident à comprendre la réalité et les pratiques; elles situent les individus dans le champ social et leur permettent d'élaborer une identité personnelle gratifiante, et de faire des comparaisons au niveau inter groupe. Par ailleurs, si les sujets ont une représentation d'une tâche particulière, ils réagiront en conséquence du point de vue cognitif. Donc, on peut affirmer que les représentations créent des attentes et des anticipations quant aux actions à réaliser et peuvent servir pour justifier les agissements. Il existe aussi une forte relation entre les représentations sociales et la culture des acteurs sociaux. Travailler avec les représentations sociales impliquent donc un travail sur l'intersubjectivité, à travers le composant affectif, culturel et cohérent que celle-ci implique pour les sujets.

Le sens de la représentation est déterminé par son contexte discursif et social, ce qui peut aider à expliquer les contradictions possibles qui peuvent surgir, d'un sujet à l'autre à l'intérieur d'un groupe ou d'un groupe à l'autre dans la même société. Par ailleurs, la construction des représentations sociales se produit à travers l'association, la généralisation et le regroupement autour de caractéristiques communes, par la transposition d'une image d'un plan à l'autre qui se focalise sur un trait particulier qui devient alors important. Elles subissent aussi le poids des influences réciproques, des négociations implicites, au cours de conversations où les personnes s'orientent grâce à des symboles, des images et des valeurs partagés et un répertoire commun d'interprétations, d'explications, de règles et procédés qui s'appliquent à la vie quotidienne. De là que Moscovici ait toujours insisté sur l'importance de réaliser une analyse du discours des sujets, des images et des métaphores qu'ils utilisent pour s'exprimer.

De même, on parle de trois dimensions des représentations : l'attitude, l'information et le champ de la représentation sociale. L'attitude est l'aspect le plus étudié en général et on la considère comme un guide de la conduite ; elle donne une orientation globale au comportement et influence la motivation du sujet en relation à l'objet de la représentation. L'information correspond à ce que les sujets savent sur l'objet de représentation et inclue d'une certaine manière les connaissances qu'exprime le sens commun. Finalement, le champ implique l'organisation hiérarchisée des contenus, des idées et des informations dans un contexte réel.

Comme l'a écrit Moscovici (1976) il est facile de capter la réalité des représentations sociales mais il l'est beaucoup moins de capter le concept. Pour comprendre comment elles se construisent, on a recours à la notion d'objectivation et à celle d'ancrage. L'objectivation permet de rendre concret ce qui est abstrait. C'est en général la première phase de construction de la représentation, au cours de laquelle on fait une sélection et on retient certains éléments de l'information disponible pour arriver à un arrangement des connaissances sur l'objet de représentation. On sort ces éléments de leur contexte et on finit par les considérer comme un reflet de choses naturelles. L'ancrage de son côté, aide à l'insertion au niveau cognitif de l'objet représenté dans le système de pensée pré-existant des sujets. Il mène à la création d'un cadre de conduite et implique la référence à une hiérarchie de normes et de valeurs. 
Finalement, mentionnons que les représentations ne sont pas figées mais qu'elles peuvent subir des transformations. Elles passent par une phase de création, de consolidation et sont sujet à des changements postérieurs. Selon que ce sont les éléments périphériques ou le noyau central de la représentation sociale, dans l'optique d'Abric, qui sont remis en cause, les modifications seront lentes, progressives ou brutales.

\section{La question de l'évaluation dans l'enseignement}

La conception et les lignes directrices de l'évaluation ont changé au cours du temps ; ne serait-ce qu'au long des dernières décennies, on s'est posé la question de décider s'il faut évaluer les savoirs ou les savoir-faire, avec des examens normatifs ou au moyen de portfolios, à partir du jugement unique du professeur ou en collaboration avec les apprenants, à l'aide de l'auto-évaluation et de l'évaluation entre pairs, etc. Par ailleurs, le fait d'évaluer peut déboucher sur des décisions plus ou moins importantes pour l'institution, le professeur et les élèves et a des implications sociales, psychologiques, culturelles et professionnelles.

Certains experts sur le sujet ont proposé des pistes intéressantes. Belair (2000) recommande de réfléchir sur pourquoi, comment on évalue, ce que cela représente pour l'évaluateur et les aspects à évaluer, afin de promouvoir le questionnement des actions et le changement des attitudes. De même, il est pertinent de mentionner qu'Aubégny (2004) affirme que «L'évaluation permet d'expliquer et de réfléchir sur les valeurs qui sous-tendent l'action et qui peuvent provoquer l'émergence et la reconnaissance de nouvelles valeurs». Ce concept nous rappelle l'idée que les représentations permettent aussi des changements dans la manière de conceptualiser les choses et d'agir. Ce même auteur (2006) soutient aussi l'idée, sous-jacente dans la vision de l'évaluation comme diagnostic, qu'elle est utile pour piloter les actions. Dans cette même ligne de pensée, Lecointe (1997) disait déjà que «l'évaluation tourne autour du manque et de l'absence, elle renvoie à l'essence et à l'existence; elle participe de la construction identitaire à partir de l'attribution ou non de valeurs». A ce sujet, nous avons aussi exposé l'importance qu'ont les représentations sociales pour la formation de l'identité individuelle à l'intérieur des groupes.

Finalement, l'évaluation génère des tensions car c'est souvent un exercice de pouvoir, qui a des répercussions au-delà des salles de classe et qui présente des facettes individuelles et collectives. On n'évalue généralement pas de la même façon, selon l'institution pour laquelle on travaille, selon si on est libre de choisir les outils d'évaluation et selon les besoins des apprenants. Traditionnellement, c'est au professeur qu'a été attribué le rôle d'évaluateur, sans donner sa place à l'élève comme on essaie de le faire ces dernières années. Cela implique que l'enseignant ait un droit de décision sur le futur de ses étudiants mais qu'en même temps il mette en jeu son image face à ses collègues et à l'institution. Est-il très sévère, trop complaisant, applique-t-il les barèmes et les consignes au pied de la lettre?

En fonction de tous les éléments exposés ci-dessus, nous partons de la présupposition que l'évaluation est un objet de représentation sociale, puisque (Moliner, 2001) elle est importante pour les sujets de la recherche car elle représente un aspect central des activités en classe; car les sujets, professeurs-élèves, élèves entre eux, professeurs-institution, interagissent réellement; car elle implique un défi, quant à la cohésion du groupe de professeurs face aux étudiants et à l'institution; car il existe des instances régulatrices, et car elle provoque des discussions et des questionnements constants. Sans aucun doute, la salle de classe fournit les conditions d'existence de représentations sociales car on peut la considérer comme un micro cosmos: il y a un contexte social formé par des acteurs, étudiants et professeurs qui apportent à cette réalité leur propre contexte, leur passé et leur vécu; il y a un échange de relations et de communications de différentes sortes; il y circulent des informations, internes et externes; il s'y produit des phénomènes d'information, de propagande et propagation, éléments essentiels pour la formation, consolidation ou transformation des représentations 
sociales, en particulier, en ce qui concerne l'évaluation dans notre travail; ceci provoque qu'au cours des semestres, l'identité sociale du professeur et de l'étudiant se construit et se modifie, aussi bien que leurs représentations sociales et leurs pratiques..

\section{La recherche}

\subsection{Le contexte}

Les sphères d'activités du CELE de l'UNAM sont très variées; on y enseigne 15 langues étrangères, à un public formé par 6000 étudiants par semestre environ, le français étant la deuxième langue en importance, après l'anglais. Le Département de Linguistique Appliquée organise les projets de recherche en pragmatique, sémantique, études interculturelles, formation de professeurs, acquisition du langage, autonomie et enseignement à distance et évaluation, entre autres centres d'intérêt. Le Cours de Formation de Professeurs est reconnu au niveau national et délivre un diplôme qui est demandé dans les institutions aussi bien publiques que privées pour pouvoir enseigner. Finalement, le CELE participe à la Maitrise en Linguistique Appliquée et au Doctorat en Linguistique de l'UNAM. Par ailleurs, depuis sa fondation il y a plus de quarante ans, le Centre joue un rôle de premier ordre dans l'évaluation des étudiants de l'Université, en ce qui concerne les langues étrangères. Sa Coordination d'Evaluation et Certification applique quelques 15000 examens par an, qui vont des examens de compréhension de lecture aux tests du DELF et aux certifications pour obtenir des bourses d' études à l'étranger.

Le Département de Français, de son côté, a toujours été à l'avant-garde de la didactique du FLE au Mexique ; il a été l'un des premiers à adopter l'approche communicative dans les années 80 et à élaborer du matériel pédagogique adapté aux étudiants mexicains. De même, il a été pionnier quant à l'organisation d'un laboratoire audiovisuel pour le travail en autonomie qui s'est ensuite cristallisé dans la création d'un centre d'apprentissage auto dirigé. Les cours sont organisés en sept semestres qui incluent la préparation aux examens du DELF ainsi que des cours consacrés uniquement à la compréhension de lecture. Il y a pratiquement 900 étudiants inscrits chaque semestre.

\subsection{La méthodologie de la recherche}

Des entretiens individuels ont été organisés, avec le support de divers outils. Tout d'abord, il a été demandé oralement aux sujets des renseignements sur leurs coordonnées personnelles : leur formation, la manière dont ils sont devenus professeurs, leur nombre d'années d'expérience, les niveaux qu'ils enseignent, le matériel qu'ils utilisent ainsi que leur relation avec leurs élèves et leur attitude vis-à-vis des langues étrangères, les francophones et hispanophones, l'enseignement et le Département de Français. Ensuite ils ont répondu par écrit à une série de questions sur leur pratique de classe où ils expliquaient comment ils évaluent leurs élèves, les éléments qu'ils prennent en considération, les qualités qu'ils apprécient chez eux, comment ils les évalueraient s'ils pouvaient ignorer les examens et les normes officiels. La troisième partie était composée de deux entretiens; le premier sur les conseils que les sujets donneraient à un professeur débutant pour qu'il puisse évaluer ses élèves et le deuxième où on leur proposait de faire des commentaires sur la pratique de l'évaluation dans le Département. Puis, ils ont réalisé un exercice d'association libre par écrit à partir du mot “évaluation" et ils ont complété la phrase "pour moi l'évaluation c'est...". Pour finir, ils ont répondu à un questionnaire sur leurs attitudes en cochant leur degré d'adhésion ou de rejet à une cinquantaine d'affirmations sur le rôle du professeur et des élèves, la culture francophone, l'enseignement, l'évaluation, etc, à l'aide d'une échelle type Likert. 
Didactique et enseignement, français langue maternelle, français langue seconde DOI $10.1051 / \mathrm{cmlf} / 2010041$

\subsection{Les sujets}

Nous avons interviewé 24 professeurs du Département de Français, dont 18 femmes et 6 hommes qui peuvent être organisés en différentes catégories : par leur nationalité, 9 natifs francophones (françaises) et 15 non natifs, par leur âge, 10 ont moins de 40 ans, et 14 plus de 40; par le nombre d'années d'expérience, 12 ont moins de 10 ans d'expérience au CELE et 10 plus de 10 ans; par leur formation, 19 ont une formation de base en Sciences Humaines et 5 en Sciences Exactes, qui vont des études de Lettres à l'Anthropologie, l'Esthétique, la Pharmacie ou la Physique; 8 professeurs ont une licence, 10 une maîtrise, 2 un doctorat; finalement, 18 sont devenus professeurs par hasard et 6 par vocation. Par ailleurs, 19 ont suivi le Cours de Formation du CELE, ou d'autres, nationaux et internationaux. Ces formations ont été suivies sur le tard dans la majorité des cas puisque 17 sujets ont dit avoir commencé à faire cours sans aucune formation en pédagogie.

\subsection{Les résultats}

Pour ce qui est de la première série de questions, concernant leurs relations avec les étudiants et leurs attitudes envers les langues étrangères, la profession et le Département, il est clair tout d'abord que leurs rapports avec leurs élèves sont privilégiés.

Ils conservent souvent l'amitié d'anciens apprenants et en parlent avec beaucoup d'affection : « je conserve leur amitié bien des semestres après »; «j'aime les accompagner dans leur processus, dans la découverte (de la langue) ».

Ils ont aussi une attitude positive envers le FLE et l'espagnol : «j'ai toujours eu la passion des langues », «l'espagnol et le français sont mes deux langues»; à l'égard des natifs des deux langues ainsi que du Département, ils ont plutôt une attitude neutre à négative : "je n'ai plus rien à faire en France », « il y a une certaine ouverture, en comparaison avec d'autres institutions ».

En ce qui concerne la profession, ils déclarent être tout à fait positifs envers l'enseignement qui « donne un sens à leur vie ». C'est une idée exprimée aussi bien par ceux qui sont devenus professeurs par hasard «la seule chose que je savais faire, c'était parler français », " quand je suis arrivée au Mexique, on m'a demandé des cours de français » que par ceux qui ont toujours senti avoir la vocation «je crois que je vais être prof toute ma vie, pour moi l'enseignement c'est une vocation », « je suis prof, à $100 \%$ ».

Leur formation leur a été très utile et leur sert encore souvent comme point de référence «ça m'a aidé à comprendre le concept de l'enseignement», « ça m'a ouvert le panorama, ça a changé ma vie », « il y a un avant et un après Formation de Professeurs ».

Il n'y a pas de différences clairement exprimées entre les sujets pris dans leur individualité, qu'ils soient natifs ou pas, âgés ou jeunes, avec beaucoup ou peu d'années d'expérience. En tant que groupe, soulignons le fait qu'il y a plus de professeurs mexicains que français et que leur niveau d'études est assez élevé : ce sont deux caractéristiques qui témoignent d'un changement par rapport à la situation d'il y a quelques années où il suffisait de savoir la langue pour être considéré capable de l'enseigner. Par ailleurs, nous pouvons tracer une identité collective de ce groupe dont les traits principaux seraient que les professeurs se plaisent à exercer leur profession car l'objet d'enseignement les passionne, parce qu'ils sentent qu'ils ont la formation adéquate pour ce faire et apprécient leurs élèves et l'institution où ils travaillent. 
$\mathrm{Au}$ sujet des représentations de nos 24 sujets sur l'évaluation, nous mentionnerons les données suivantes obtenues du croisement des différents outils que nous avons utilisés, les chiffres entre parenthèses représentant le nombre de sujets ayant répondu.

Le trait fondamental de l'évaluation est d'être un processus (21 sujets), un produit (2) : «il faudrait mieux voir le processus que le produit», «l'évaluation comme note finale ou l'évaluation comme prise en considération de ce que l'élève fait pendant tout le semestre? ». Un seul autre sujet a répondu qu'il était indécis entre les deux options.

Cependant, ce qui est intéressant à remarquer, c'est que tous nos sujets sont en général d'accord sur d'autres caractéristiques de l'évaluation, comme le fait que c'est : un bilan (11), utile pour professeurs et élèves (13) : « il arrive un moment où je ne sais plus où j'en suis, parce que c'est aussi le problème du professeur, alors je fais une petite évaluation pour voir s'ils ont assimilé ce que j'ai fait ».

Elle doit être : continue (16) : «essayer d'évaluer ce qu'ils ont appris à la fin, mais d'abord au milieu du semestre, petit à petit faire des évaluations, l'évaluation continue, c'est la meilleure », formatrice (8) : « il faut voir les deux aspects de l'évaluation, l'évaluation formatrice et l'évaluation sommative qui lui permet de passer, mais surtout la formatrice où le professeur peut intervenir sur les progrès des élèves, où l'élève peut intervenir su sa propre formation », cohérente avec ce qui est fait en classe (6) : «si tu as travaillé certaines choses en classe, c'est ça qu'il faut évaluer en premier ».

Elle se base sur la coresponsabilité professeur/élèves (23) : « le professeur n'a pas toute la responsabilité vis à vis des élèves, il faut qu'ils se rendent compte qu'ils ont une tâche fondamentale dans l'apprentissage de chacun à l'intérieur du groupe », sur une augmentation de l'exigence selon le niveau d'étude de FLE (9), sur l'engagement personnel de l'élève (8).

Elle porte sur une séquence d'apprentissage (15), les quatre compétences (8) : « je recommanderais de ne rien oublier, d'essayer d'évaluer les quatre compétences », la capacité de communication (8), les progrès des élèves (6). Elle se sert de l'autoévaluation (24), de feed back à l'élève (22), d'une panoplie d'outils (14) : « pour évaluer il n'y a pas que les examens, il y a de nouvelles tendances, il faut avoir d'autres paramètres, tenir compte des devoirs, de leur participation, pour qu'ils arrêtent de demander l'examen », de négociations (6) mais n'a pas besoin de notes ou d'examens (20): «prendre en considération tout ce qui a été vu pendant le semestre, si les élèves avancent, leur autoévaluation ».

Si l'on examine les opinions des professeurs au sujet des examens élaborés au niveau du Département, à raison d'un examen semestriel par niveau comme évaluation finale, on trouve les commentaires suivants. Les examens ne sont pas adéquats, ni aux programmes, ni aux objectifs, ni aux contenus des cours ni à la réalité de la salle de classe (14); le fait qu'il y ait un seul examen officiel est négatif (10); les examens sont toujours les mêmes (9), leur degré de difficulté n'est pas adéquat (9), ils présentent des erreurs de syntaxe, d'orthographe (8), n'offrent pas d'activités significatives (7). Les examens sont plus ou moins corrects (2). Les opinions exprimées sont en général tranchantes : «on veut nous faire évaluer des choses qui ne sont pas au programme », « ils sont tellement faciles que tout le monde réussit », « c'est toujours le même examen, avec les mêmes erreurs ».

Finalement, si on analyse les commentaires sur l'utilité des examens du Département en relation aux pratiques d'évaluation réelles des professeurs on arrive à la conclusion que : l'évaluation que réalisent les professeurs à partir de leurs propres outils leur semble plus importante que les examens officiels (20); ils appliquent l'examen officiel parce que c'est pratiquement obligatoire ou parce que les élèves le leur demandent, mais ne l'apprécient pas (17); ils adaptent l'examen, en retirent des parties, en mettent d'autres (11); ils appliquent l'examen officiel sans problèmes (3). Un seul sujet affirme que pour lui tous les outils, les siens et l'examen 
départemental, ont la même importance. Par ailleurs, ils apprécient particulièrement les élèves qui sont curieux intellectuellement, constants dans leur apprentissage et qui osent poser des questions.

Il nous semble important de souligner le fait que tous les résultats coïncident, autant dans les réponses aux questions ouvertes et fermées que dans l'exercice d'association libre. En effet, dans celui-ci, les sujets ont mentionné en premier lieu les mots apprentissage, processus, bilan et stress. Certains sujets avaient dit au cours des entretiens que quand on parle d'évaluation, on pense immédiatement à l'angoisse, par exemple. L'association libre permet de voir dans notre cas qu'effectivement l'évaluation est perçue non seulement comme source de tension mais aussi comme une action qui va vers le diagnostic, la formation, fondés sur différents instruments et dont l'objet est l'apprentissage des étudiants.

\section{Métaphores et discours}

Les représentations sociales sont difficiles à cerner ; car les sujets étudiés peuvent craindre de perdre la face, quand ils donnent leur opinion et s'expriment devant le chercheur et donc il existe toujours une marge de doute quant à savoir si ce qui a été dit reflète réellement ce qui est pensé, ou fait. Une des manières de suivre la piste de leurs représentations est d'analyser les métaphores et le discours des sujets.

\subsection{Les métaphores}

Dans notre cas, il est intéressant de remarquer que, même si les sujets n'ont pas utilisé un grand nombre de métaphores, celles que nous avons recueillies vont dans le même sens que les résultats que nous avons exposés ci-dessus. En effet, l'idée de processus se retrouve dans les images que les sujets ont exprimées: «Nous allons dans le même sens »; « les élèves montent dans un bateau et nous devons les emmener à bon port »; «j'aime bien mettre les élèves sur les rails »; l'idée du voyage implique un processus, avec un départ, un parcours et une arrivée. De même, un autre sujet a parlé de l'évaluation comme « un fruit qui arrive à maturation » où l'on peut penser à la graine qui est plantée, grandit et mûrit au cours d'un processus plus ou moins long. Mais les aspects négatifs de l'évaluation, qui avaient été mentionnés comme le stress et l'angoisse, apparaissent aussi car on trouve l'image de "évaluer, ce n'est pas faire passer à la guillotine » mais aussi «les élèves ne doivent pas expier un péché». Il est curieux de voir que plusieurs sujets ont commenté que «le professeur ne peut pas tout savoir parce qu'il n'est pas Dieu »; cela nous permet de penser que les erreurs, les manques, l'ignorance sont acceptés. Ceci est aussi un commentaire important si l'on se rappelle que pendant longtemps dans la pensée pédagogique, la faute était inacceptable. Finalement, l'évaluation qui doit être formatrice et adaptée aux groupes ou à ce qui a été vu en classe «doit être comme un costume sur mesure ».

Ces quelques exemples montrent bien une image objectivée de l'évaluation qui la rend plus accessible aux sujets, et où l'idée de processus est reprise plusieurs fois de manière très vivante.

\subsection{Le discours}

Finalement, nous avons réalisé une analyse du discours des sujets, surtout dans la partie des conseils et des commentaires où ils pouvaient s'exprimer librement. Celle-ci révèle bien le poids social, culturel et affectif dont est chargée l'évaluation. Le discours a été produit dans une situation qui pouvait provoquer une certaine tension chez les locuteurs et qui a sûrement influencé leur manière de s'exprimer, de modaliser leur pensée, de chercher une certaine cohérence et de vérifier l'efficacité de la communication avec l'interlocuteur. En 
effet, dans un premier temps, les sujets hésitent beaucoup à répondre. Ils répètent et reformulent les questions, ils rient, utilisent beaucoup de formules de remplissage; il est évident qu'ils se sentent un peu mal à l'aise, et ne savent pas comment répondre, au cas où leurs opinions les compromettraient. Ils émettent un avis à l'emporte-pièce, puis donnent des exemples qu'ils tirent de leurs propres expériences en classe et reprennent de l'assurance. Pour les conseils, ils vont du général au particulier et recréent souvent la réalité de la classe : « alors je leur dis de faire l'exercice et puis [ dîtes-moi comment vous trouvez ce que vous avez fait, comment vous évaluez ce travail ?] ».

Quand ils font des commentaires sur le Département, on perçoit clairement que c'est encore plus difficile pour eux de donner leur opinion. Certains soulignent le fait qu'ils ne parlent pas beaucoup avec leurs collègues et ne savent pas où ils en sont, alors que d'autres font des critiques féroces : "il est évident qu'il faut changer les examens », " c'est le désordre absolu ». Il semblerait qu'ils finissent par dire ce qu'ils pensent car leur degré de mécontentement et leurs questionnements sont très forts.

Les sujets utilisent constamment un métadiscours pour exprimer leurs opinions et protéger leur image. Ils reformulent et font des paraphrases, posent des questions à l'intervieweur auxquelles ils répondent immédiatement eux-mêmes. Cependant on remarque qu'ils organisent leur discours de façon cohérente avec des expressions comme « « peu à peu », " au début...ensuite». Ils ont donc apparemment une vision articulée des actions, des attitudes à assumer vis-à-vis de l'évaluation, bien qu'ils ressentent des difficultés à verbaliser leurs idées.

Les actes de parole qui sont les plus fréquemment présents sont : expliquer, convaincre, critiquer, comparer, donner une opinion, prévoir et se plaindre. Ils constatent et décrivent la situation du Département, prévoient l'évolution probable que peut subir le Centre au long des prochaines années, jugent son état actuel et donnent leur opinion.

Par ailleurs, ils ont recours à différentes modalisations, assertive et injonctive, déontique et appréciative pour présenter leurs positions face aux thèmes abordés, assumer la responsabilité de ce qu'ils disent ou préciser obligations et devoirs d'enseignement. Ainsi il y a une grande fréquence d'adverbes comme «vraiment», « réellement », « absolument », des verbes comme « je considère que », « je crois », "j'ai l'impression que », des expressions comme «à mon avis », « selon moi », où l'utilisation de la première personne du singulier rend encore plus évidente l'implication du locuteur.

On distingue aussi facilement une utilisation du conditionnel comme temps verbal, assez logique dans cette situation de communication, où les sujets essaient de prévoir, proposer des actions possibles ou recommandables : "je dirais que.. », « je préfèrerais que...», « je proposerais », « il serait nécessaire...». On se trouve devant des sujets qui nuancent leur pensée pour, peut-être, ne pas paraître trop durs ou trop critiques vis-à-vis de l'institution mais en même temps, ils sont modestes en relation à comment ils font les choses, eux-mêmes : «je pourrais donner des exemples de ce que je fais moi, bon, même si ce n'est pas parfait». Cependant, même si on remarque qu'il y a beaucoup d'hésitations et de rires tout au long de leur discours, on trouve aussi des expressions comme « je suis sûr que », « il est logique que » « tu ne vas pas faire ça quand même » quand ils expliquent ce qu'ils font eux, et qui leur a donné de bons résultats.

Par ailleurs, il est clair qu'ils ont une idée bien définie sur ce qu'il est possible ou obligatoire de faire. En effet on trouve les verbes « pouvoir » et « devoir » conjugués au présent et au conditionnel, à la première personne du singulier et du pluriel et à la deuxième du singulier . Ceci est renforcé par des expressions comme «il est essentiel que », « là, c'est obligatoire », « tu es là pour... ».

Pour insister sur leur point de vue, s'affirmer et réitérer en quelque sorte qu'ils ont raison et qu'ils savent de quoi ils parlent sur un sujet si délicat, ils utilisent beaucoup d'adjectifs et d'expressions : "préférable », 
«incroyable », « normal », « important », « difficile », « impossible », « rien d'extraordinaire » ou bien « c'est sérieux », «c'est valable », « on traîne des contradictions », « à tout prix ». De plus, on trouve une grande quantité de «très », « tout », « beaucoup »: «très problématique », « très mauvais », « toute une feuille de... », « tout le temps », « je fais beaucoup...».

Ils se démarquent et insistent bien sur la différence entre ce qu'ils font, et ce que font les autres ou le Département comme instance officielle; pour ce faire, ils utilisent beaucoup d'oppositions : «moi....les collègues », « moi...les élèves », mais aussi « on....le Département », où l'on perçoit une certaine solidarité d'équipe mais aussi une conscience claire de la position de chacun. Ils utilisent beaucoup de négations et d'antonymes, comme si au long de leur discours ils voulaient rejeter certains concepts, comparer, juger et donner un panorama plus vaste de la situation : «si tu ne fais pas pression, ils ne font rien », " pas seulement la grammaire », « ce n'est pas utopique, c'est possible », « un professeur sans formation mais qui a de l'expérience ». Il est intéressant aussi de remarquer qu'ils se situent dans leur contexte de travail, où justement se manifestent leurs attitudes et leurs pratiques à partir de leurs représentations, " ici », "à l'Université », « maintenant », « avant ».

\section{Conclusion}

Des tendances claires et assez cohérentes apparaissent tout au long des résultats. Nous mentionnerons l'attitude positive envers la profession et le plaisir d'enseigner; les bonnes relations avec les étudiants, la langue française et la culture francophone, objet de la profession; les sujets se sentent à l'aise dans leur institution et cela est important car les représentations, comme nous l'avons vu, sont inscrites, naissent et se forment dans des contextes sociaux particuliers. Les caractéristiques de l'UNAM et du CELE influencent sûrement sur les représentations et les pratiques des professeurs : l'institution publique la plus importante du pays, à l'avant-garde des innovations, où la liberté d'enseignement est une valeur fondamentale ainsi que la formation continue du corps enseignant.

Evaluer implique une pression, des éléments cognitifs, psychologiques et sociaux et les résultats de notre travail montrent que les professeurs de FLE du CELE ont une représentation sociale de l'évaluation comme un processus, qui s'oriente vers le bien-fondé d'un travail qui doit être continu, formatif, et qui a une large vision, car on ne juge pas seulement les paramètres linguistiques mais aussi les éléments culturels sans besoin de notes ou d'examens; de même ils apprécient clairement la valeur de l'exigence du point de vue académique, de l'auto évaluation, la coresponsabilité et le retour au sujet qui aident autant le professeur que l'élève au cours du processus d'enseignement apprentissage.

Nous avons pu détecter que les sujets ont des attitudes positives et ouvertes envers l'évaluation, même si le thème provoque des tensions et si évaluer est perçu comme quelque chose de difficile et problématique, bien que nécessaire et utile, par ailleurs.

Les sujets sont bien informés et ont des connaissances diverses sur les moments, les outils, les buts et les fonctions de l'évaluation.

De même, nous avons donc trouvé un champ de représentations structuré et cohérent : on distingue le quand, pourquoi, qui, quoi, comment de l'évaluation à travers le discours et les réponses des sujets.

Nous nous trouvons face à une pratique solide, de sujets informés et qui expriment une représentation en accord avec les tendances en didactique actuelle. Par contre, il n'apparaît pas de différences claires entre les 
différentes catégories de sujets (en fonction de leur âge, années d'expérience, formation ou origine) et l'on peut penser qu'il s'agit d'un groupe qui a élaboré une représentation qui recouvre un consensus, au cours de leurs années d'expérience, de formation en commun, de discussions et de travail en équipe.

Ils ont une position nettement définie contre les modalités d'évaluation proposées par le Département qui donne l'impression de ne pas arriver à être à la hauteur des changements et des exigences actuels. Peut-être cela est-il dû au fait que les programmes ont subi plusieurs changements au cours des derniers semestres et l'élaboration des examens n'a pas pu en suivre le rythme.

Les pratiques des sujets sont cohérentes avec ce qu'ils ont exprimé quant à leur représentation sur l'évaluation et on peut en conclure à une influence mutuelle où l'adaptation aux besoins des élèves dans le cadre de certaines normes de références et les apports de nouvelles connaissances amènent des changements en douceur dans le dire, le faire et le penser.

Nous pouvons donc affirmer qu'au cours de cette recherche nous avons pu détecter des attitudes manifestées clairement par les sujets, des informations variées et complètes exprimées sur l'évaluation qui finalement aident à constituer un champ de représentation sociale sur l'évaluation dans le contexte précis du Département de Français du CELE de l'UNAM. Il serait intéressant dans le futur de faire la même recherche dans d'autres Départements, ou avec les étudiants, qui sont finalement les sujets des activités d'évaluation.

\section{Bibliographie}

Abric, J.C. (1994). Pratiques sociales et représentations. Paris: PUF.

Aubégny, J. \& L. Clavier (2004). L'évaluation en IUFM. Paris : L'Harmattan

Aubégny,J, \& L. Clavier (eds) (2006). L'évaluation. Entre permanence et changement. Paris: L'Harmattan

Banchs, M.A. (2000). Aproximaciones procesuales y estructurales al estudio de las representaciones sociales. Papers on Social Representations, 9, 3.1-3.15

Bélair, L. (2000). La evaluación en la acción. Sevilla: Diada

Castorina, J.A. (2008). Representaciones sociales. Problemas teóricos y conocimientos infantiles. Barcelona: Gedisa

Doise, W. \& A. Palmonari (1986). L'étude des représentations sociales. Neuchâtel : Delachaux et Niestlé

Figari, G. \& M. Achouche (2001). L'activité évaluative réinterrogée. Bruxelles : De Boeck Université

Grize, B. (1996) Logique naturelle et communication. Paris : PUF

Ibañez. T. (1988). Ideologías de la vida cotidiana. Barcelona : Sendai

Jodelet, D. (1999). Les représentations sociales. Paris: PUF.

Lecointe, M. (1997). Les enjeux de l'évaluation. Paris : L'Harmattan

Marková, I. (2003). Dialogicality and Social Representations. Cambridge :CUP

Moliner, P. (2001). La dynamique des représentations sociales. Grenoble : PUG

Moliner,P, P. Rateau \& V. Cohen-Scali (2002). Les représentations sociales. Pratiques des études de terrain, Rennes : PUR

Moore, D. (coord) (2005). Les représentations des langues et de leur apprentissage. Références, modèles, données et méthodes. Paris : Didier 
978-2-7598-0534-1, Paris, 2010, Institut de Linguistique Française

Didactique et enseignement, français langue maternelle, français langue seconde DOI $10.1051 / \mathrm{cmlf} / 2010041$

Moscovici, S. (1961). La psychanalyse, son image, son public. Paris : PUF

Moscovici, S. (1972). Introduction à la psychologie sociale. Paris: Larousse

Moscovici, S. \& Farr R. (1984). Social Representations. Cambridge: CUP

Pereira de Sá, C. (1998). A construçao do objeto de pesquisa em representaçoes sociaes. Rio de Janeiro: ed UERJ

Piña, J.M. \& Y. Cuevas (2004). La teoría de las representaciones sociales. Su uso en la investigación educativa en México.Perfiles Educativos, 105-106, 102-124

Wagner, W. \& F. Elejabarrieta (1996) Representaciones sociales. In Morales, F. Psicología Social. México: Mc Graw Hill. 814-842. 